\title{
Effect of leg weight shifting on muscle activation of the trunk and lower extremity during trunk flexion and extension performance
}

\author{
Suyoung Shim ${ }^{\mathrm{a}}$, Yijung Chung ${ }^{\mathrm{b}}$ \\ ${ }^{a}$ Department of Physical Thaerpy, Uijeongbu Healings Hospital, Uijeongbu, Republic of Korea \\ ${ }^{b}$ Department of Physical Therapy, College of Health Science and Social Welfare, Sahmyook University, Seoul, Republic of Korea
}

Objective: To investigate the effect of performing three different toe touch (TT) task condition on the activities of four different muscles using surface electromyography (sEMG) in healthy young adults.

Design: Cross-sectional study.

Methods: A total of 20 healthy young adults ( 6 males, 14 females) voluntarily participated in this study. All subject randomly performed three different TT task conditions as follows: general toe-touch (GTT) task, one side toe touch (TT) task during weight bearing, and one side foward toe touch (FTT) task during weight bearing. The muscle activities of erector spinae (ES), gluteus maximus (GM), hamstring (HAM), tibialis anterior (TA) muscles during the TT task were measured using sEMG. Subject performed each of the three conditions three time in random order and mean values were obtained.

Results: With the trunk flexion period, the TT and FTT showed significantly greater muscle activity in the GM, HAM and TA compared to the GTT task $(p<0.05)$. The TT position showed significantly greater HAM muscle activity than the GTT position. The dominant and nondominant ES muscle activity was significantly greater in the FTT compared to the GTT position $(p<0.05)$. The dominant GM, HAM, and TA was significantly greater in the TT and FTT compared to the GTT position $(p<0.05)$. Although the dominant ES was significantly greater in the TT and FTT compared to the GTT position $(p<0.05)$, the dominant GM muscle activity was signifcantly greater in the TT compared with the GTT position $(p<0.05)$.

Conclusions: These findings may be applicable within the clinical field for selective trunk and lower extremity muscle activation and basic biomechanics purpose.

Key Words: Electromyography, Lower extremity, Weight bearing

\section{Introduction}

Low back pain associated with upper body and hip neuromuscular activation patterns is experienced in $80 \%$ of the general population during their lifetime. Low back pain is the most common musculoskeletal disorder and is referred to as a misalignment syndrome accompanied by pain and joint dysfunction due to asymmetric body imbalance of muscles, joints, and range of motion [1]. Back pain may occur due to having a lesion in the lumbar region or lumbar spine that is associated with the lumbar pelvic rhythm [2]. In particular, the working posture, which requires continuous upper body flexion and extension, is one of the causes and known to be associated with back pain [3-5].

The actual mechanisms involved with back pain are not clearly stated, and clinical and neurological diagnostic tests for back pain are also unclear. Dynamic electromyography (EMG) is used to assess the physical ability of patients with back pain [6]. A dynamic study of EMG signal analysis has been conducted to evaluate the mechanism of lumbago, and

Received: 27 February, 2018 Revised: 13 March, 2018 Accepted: 13 March, 2018

Corresponding author: Yijung Chung (ORCID http://orcid.org/0000-0002-2431-8895)

Department of Physical Therapy, College of Health Science and Social Welfare, Sahmyook University, 815 Hwarang-ro, Nowon-gu, Seoul 01795, Republic of Korea

Tel: 82-2-3399-1637 Fax: 82-2-3399-1639 E-mail: yijung36@syu.ac.kr

(c) This is an Open-Access article distributed under the terms of the Creative Commons Attribution Non-Commercial License (http://creativecommons.org/licenses/ by-nc/4.0) which permits unrestricted non-commercial use, distribution, and reproduction in any medium, provided the original work is properly cited.

Copyright $\odot 2018$ Korean Academy of Physical Therapy Rehabilitation Science 
epidemiological studies have been carried out to evaluate the activity of the trunk muscle and the differences in exercise patterns between normal and low back pain patients [7]. Recently, a study on muscle activity and motion pattern through dynamic tasks has been reported [2]. Leinonen et al. [8] investigated how the dynamic mobilization patterns of the lumbar and hip joint extensors during trunk flexion-extension differ from those of normal subjects. Hodges and Richardson [9] studied the changes in order of mobilization of trunk muscles. In addition, Shan et al. [10] and others have been conducting various studies on muscle activity and movement patterns through dynamic tasks such as studying the onset time through flexion to extension.

Previous studies on muscle activity, muscle initiation time, and muscle relaxation through general toe touch (GTT) tasks have been actively conducted, but studies that include and apply environmental factors that may influence trunk extension are lacking.

Therefore, the purpose of this study was to investigate the effects of trunk flexion and extension on the body and lower extremity muscles in healthy normal adults, to determine the difference in the activity of the trunk muscles according to the weight support environment, and to provide basic data on the evaluation of the injuries and diseases that may cause changes in back and lumbar pelvic rhythm.

\section{Methods}

\section{Subject}

Subjects from Healings Hospital in the Uijeongbu area who provided their informed consent and voluntarily agreed to participate were included in the study.

Twenty healthy subjects who met the selection criteria were randomly selected to minimize bias. Six men and fourteen women who did not participate in extensive exercise a week before the experiment were included. Exclusion con-

Table 1. General characteristics of the subjects

$(\mathrm{N}=20)$

\begin{tabular}{lc}
\hline Characteristic & Subject \\
\hline Sex & \\
Male & 6 \\
Female & 14 \\
Age $(\mathrm{y})$ & $25.70(2.69)$ \\
Height $(\mathrm{cm})$ & $167.40(7.57)$ \\
Weight $(\mathrm{kg})$ & $63.20(9.57)$ \\
\hline
\end{tabular}

Values are presented as number only or mean (SD). ditions included those with congenital anomalies in the upper and lower limbs, those with neurological and skeletal muscle defects, those with medically diagnosed back and leg injuries, those diagnosed with medical problems, those with musculoskeletal pain, those who have no abnormalities in the total range of motion of the trunk forward flexion, and those who have consumed alcohol or caffeine on the day before and day after the experiment. All subjects had no other chronic diseases or disease associated with the erector spinae (ES), gluteus maximus (GM), hamstring (HAM), and tibialis anterior (TA) muscle (Table 1). In this study, the subjects were fully informed about the experimental conditions and procedure prior to the experiment and signed the consent form of agreement. The study was approved by the research ethics committee of Sahmyook University (Approval No. 2-1040781-AB-N-01-2017104HR).

\section{Procedures}

The maximum voluntary isometric contraction (MVIC) was measured after the electrodes were attached to the ES, GM, HAM, and TA muscles. Prior to the experiment, the researchers explained to the subjects about the touch toe task, and each participant practiced each position three or more times to understand the experimental method.

The TT task was performed as follows, which was repeated three times and was performed in a random order.

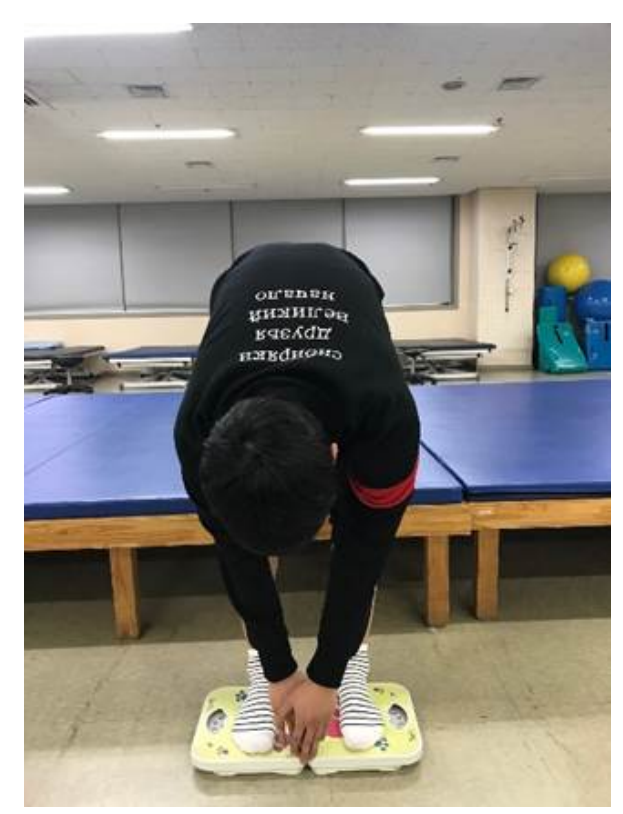

Figure 1. General toe touch task. 


\section{General toe touch (GTT) task position}

Starting from a straight standing posture with legs shoulder-width apart and the knees straight, at the start of the researcher's instructions, the subject performed trunk flexion until the tip of the middle finger touch their toes, and trunk extension was performed until their shoulders passed over the heels [11] (Figure 1).

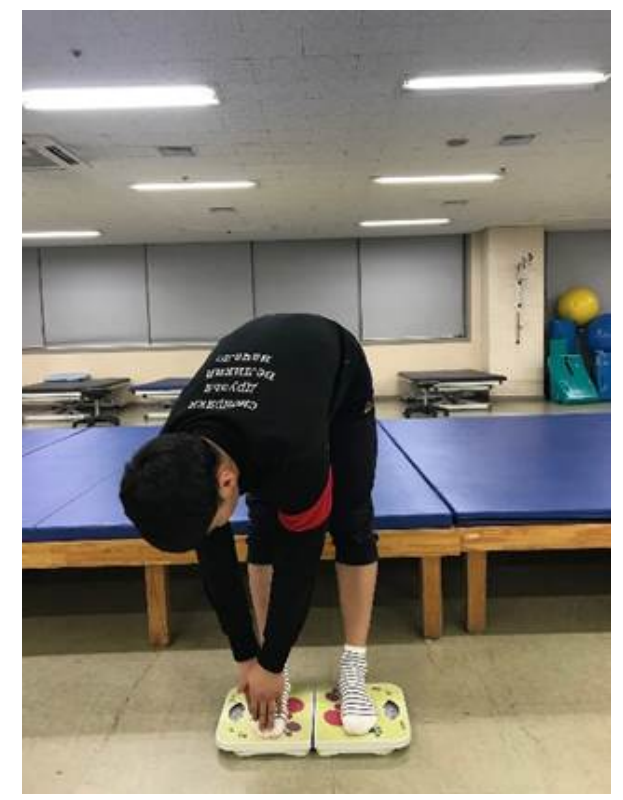

Figure 2. One side toe touch task during weight bearing.

\section{One side toe touch (TT) task during weight bearing}

Starting from the general TT task position and after the body weight has been shifted onto the dominant side, at the start of the researcher's instructions, the subject performed trunk flexion until their middle finger touched the tips of the toes and performed trunk extension until the shoulder passed over the heels (Figure 2).

\section{One side forward toe touch (FTT) task during weight bearing}

Starting from the general TT task position, the lower extremities were spread apart with the dominant lower limb placed anteriorly and knees fully extended. At the signal of the researcher, the subject performed trunk flexion until the middle finger touched the tips of their toes and shoulder extension was performed until the trunk passed over the heels (Figure 3).

The on side TT task during weight bearing and one side FTT task during weight bearing postures refer to the support of more than $55 \%$ of the body weight [10]. Each condition was repeated three times, and trunk flexion and extension was maintained for 5 seconds during the TT task posture. Subjects were allowed to rest for 1 minute between each condition, and measurements were taken during the random performance of each posture that was performed three times.
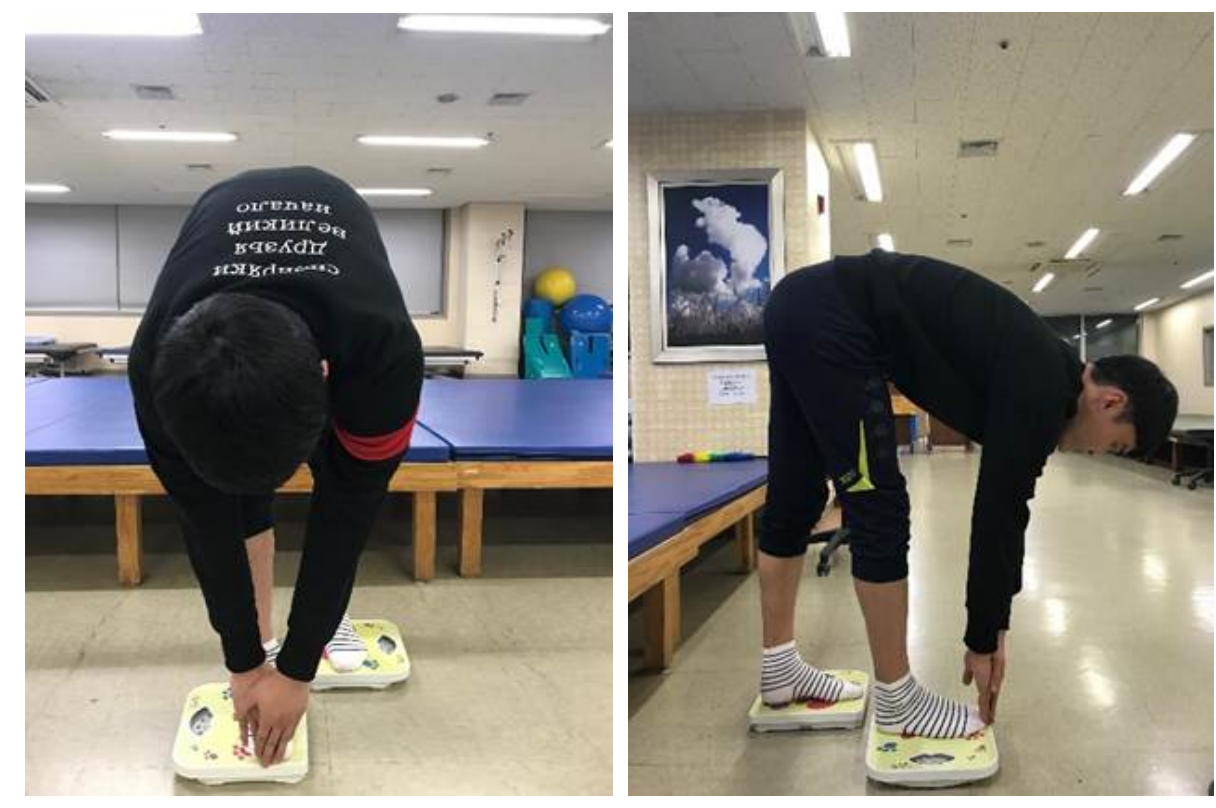

Figure 3. One side forward side toe touch task during weight bearing. 


\section{Measurement}

\section{Collection of EMG materials}

To assess muscle activity during the TT task position, the surface EMG Telemyo 2400 G2 Telemetry EMG system (Noraxon Inc., Scottsdale, AZ, USA) was used. The sampling rate of the EMG signal was set to $1,000 \mathrm{~Hz}$, and the frequency bandwidth was set to 20 to $500 \mathrm{~Hz}$. The EMG signals measured in this study were obtained using the MyoResearch XP Master Edition 1.07 XP software (Noraxon Inc.) and the root mean square value of $50 \mathrm{~ms}$ was obtained after full wave rectification. Electromyogram signals were collected for a total of 5 seconds, with data extracted from the middle $3 \mathrm{sec}$ onds and the first and last second excluded. The extracted EMG signal was normalized to the \%MVIC value obtained by measuring the EMG signal three times in total and converting the average of the EMG signals into a percentage of the mean square root of the MVIC of each muscle. The $\% \mathrm{MVIC}$ indicates that all the data represent a percentage between $0 \%$ and $100 \%$ and can normalize the function of the muscles and enable individual comparisons of each muscle [12].

The surface electrode for the ES was placed $2 \mathrm{~cm}$ laterally from the third lumbar spinous process and parallel to the iliac crest. In the case of the GM, the electrode was placed in the muscle belly at the center at the line between the lateral angle of the sacrum and greater trochanter. For the medial HAM (semimembranous), the electrodes were placed $15 \mathrm{~cm}$ inferior to the ischial tuberosity. For the anterior TA, the electrodes were placed parallel to and laterally along the central axis of the tibia. To measure the MVIC, the muscle contraction values of the ES, GM, medial HAM, and TA muscles were measured for 5 seconds before each experiment. Each muscle was measured by the same assessment position used with the manual muscle test [12]. For the MMT measurement position for the ES, the subject laid in prone position and lifted their trunk while a therapist stabilized the subject's lower extremities. The GM was examined with the subject lying in prone position, and with the therapist stabilizing the upper limbs, the subject lifted one lower extremity. For the HAM, the subject laid in prone position and performed knee flexion while the therapist stabilized the femur. For the TA, the subject was in sitting position and performed ankle dorsiflexion while the therapist stabilized the tibia.

\section{Data and statistical analysis}

The collected data were analyzed using the PASW Statistics ver. 19.0 (IBM Co., Armonk, NY, USA). The general characteristics of the subjects were analyzed by descriptive statistics and one-way repeated measures analysis of variance was conducted to investigate the effect of performing toe touch task on trunk and lower extremity muscle activity. The least significant difference was used for post-test analysis to determine whether there was a difference in the muscle activity between the trunk and lower extremities. All data were statistically significant at $p<0.05$.

\section{Results}

The muscle activity during the toe touch task posture according to weight bearing are as follows (Table 2).

Table 2. Comparison of muscle activity according to 3 conditions

$(\mathrm{N}=20)$

\begin{tabular}{|c|c|c|c|c|c|c|c|c|}
\hline \multirow{2}{*}{ Condition } & \multicolumn{2}{|c|}{ ES } & \multicolumn{2}{|c|}{ GM } & \multicolumn{2}{|c|}{ HAM } & \multicolumn{2}{|c|}{ TA } \\
\hline & Right & Left & Right & Left & Right & Left & Right & Left \\
\hline \multicolumn{9}{|l|}{ Flexion period } \\
\hline GTT (\%MVIC) & $24.96(2.85)$ & $23.99(2.52)$ & $9.67(1.48)$ & $9.72(2.37)$ & $10.48(1.22)$ & $9.71(1.31)$ & $3.80(0.82)$ & $5.61(1.51)$ \\
\hline TT (\%MVIC) & $27.54(2.65)$ & $24.33(2.91)$ & $16.37(1.94)^{*}$ & $5.77(1.41)$ & $18.03(1.62)^{*}$ & $7.67(1.28)^{*}$ & $12.55(3.23)^{*}$ & $2.43(0.47)$ \\
\hline FTT (\%MVIC) & $31.02(3.24)$ & $25.45(3.26)$ & $16.37(1.53)^{*}$ & $8.65(2.42)$ & $19.72(1.85)^{*}$ & $9.69(1.64)$ & $17.69(4.08)^{*}$ & $5.13(1.43)$ \\
\hline \multicolumn{9}{|l|}{ Extension period } \\
\hline GTT (\%MVIC) & $50.11(3.53)$ & $48.31(3.31)$ & $13.89(2.22)$ & $13.24(2.11)$ & $13.42(1.41)$ & $13.12(1.56)$ & $6.66(1.97)$ & $7.25(1.61)$ \\
\hline TT (\%MVIC) & $54.91(4.20)$ & $60.60(4.36)^{*}$ & $20.03(2.25)^{*}$ & $6.83(1.38)^{*}$ & $20.54(2.56)^{*}$ & $13.93(1.69)$ & $15.57(3.12)^{*}$ & $3.93(0.96)$ \\
\hline FTT (\%MVIC) & $59.50(4.45)^{*}$ & $63.07(4.86)^{*}$ & $22.39(2.72)^{*}$ & $9.54(1.96)$ & $25.28(2.87)^{*}, \dagger$ & $14.84(1.93)$ & $20.38(4.43)^{*}$ & $4.56(1.41)$ \\
\hline
\end{tabular}

Values are presented as mean (SD).

ES: erector spinae, GM: gluteus maximus, HAM: hamstring, TA: tibialis anterior, GTT: general toe touch task, TT: one side toe touch task during weight bearing, FTT: one side forward toe touch task during weight bearing.

${ }^{*}$ Significant difference compared with GTT $(p<0.05)$. ${ }^{\dagger}$ Significant difference compared with TT $(p<0.05)$. 


\section{Comparison of muscle activity during the one side TT task during weight bearing in the trunk flexion period}

The TT and FTT tasks generated significantly greater muscle activity of the GM, HAM, and TA compared to the GTT position task $(p<0.05)$.

\section{Comparison of muscle activity with the one side TT task during weight bearing posture during the trunk extension period}

Although the dominant and non-dominant ES muscle activity was significantly greater in the FTT condition compared to the GTT $(p<0.05)$, the muscle activities of the dominant GM, HAM, and TA were significantly greater in the TT and FTT positions compared with the GTT position $(p<0.05)$.

In addition, the dominant HAM muscle activity was significantly greater in the FTT compared to the TT position $(p<0.05)$. Although the dominant ES was significantly increased in the TT and FTT posture compared to the GTT $(p<0.05)$, the dominant GM muscle activity was significantly greater in the TT compared with the GTT posture $(p<0.05)$.

\section{Discussion}

The purpose of this study was to investigate the changes in muscle activity of the ES, GM, HAM, and TA muscles of healthy persons during the TT task position with trunk flexion and extension.

The results of this study showed that TT and FTT toe task positions produced significantly greater GM, HAM, and TA muscle activity of the dominant side compared to the GTT task position. In addition, during the extension of the trunk, the TT and FTT task positions produced significantly greater muscle activity in the GM, HAM, and TA of the dominant side compared with the general touch the toe posture $(p<0.05)$.

According to Jo [13], the muscle activity of the gluteus medius, rectus femoris, biceps femoris, medial gastrocnemius, and tibialis muscle were significantly increased with weight bearing. Boren et al. [14] also reported that the muscle activity of the GM and gluteus medius were greater in the weight bearing environment compared to non-weight bearing. Studies by Bolgla and Uhl [15] also showed that the muscle activity of the gluteus medius muscle was significantly increased during hip joint motion with full weight- bearing compared to partial weight bearing. In addition, when the weight bearing was increased, the stability of the periarticular ligaments increased due to the increased pressure of the joint [16].

The results suggests that in the weight bearing condition, greater momentum is generated than in the partial weightbearing condition, requiring greater muscle activity to maintain balance [17]. Therefore, it is considered that muscle activity is increased with increasing weight bearing.

Based on the results of this study, the TT task position produced significantly greater muscle activity in the dominant HAM compared to the GTT task position. In addition, the FTT task position produced a significantly greater amount of activity in the dominant HAM compared to the TT task position $(p<0.05)$.

According to Prince et al. [18], due to the shift in the center of gravity, a strategy for adjusting balance is required for the forward weight bearing position more so than with the lateral weight bearing position.

In addition, it is more difficult to maintain balance in the diagonal stance because it requires a complete transfer of weight.

This result shows that the increase of instability due to the forward movement of the pressure center point has increased the muscle activity to overcome this [19]. The significant increase in HAM muscle activity is considered to be due to the role of leverage. One of the functions of the lever is to convert the force into torque, and the larger the distance from the center axis, the higher the torque [20]. Therefore, the exhibition of greater muscle activity with the FTT position compared to the TT position, and with TT compared to the GTT task position is considered to be due to an increasing distance from the center axis from the hip joint, and thus a longer lever arm, which generates greater force and muscle activity.

In addition, studies by Woo et al. [21] have reported that the activity of the gastrocnemius and TA muscles increases mainly when various proprioceptive sensory stimuli are provided for balance. It is considered that the significant increases in the lower extremity extensor and TA muscle activity were due to weight bearing and for the sake of maintaining balance.

The results of this study showed that the TT and FTT position during the trunk extension period showed a significantly greater increase in non-dominant ES activity compared to the general TT task position.

Park et al. [22] reported that in the normal adult, the mus- 
cle activity was significantly elevated in the contralateral multifidus, ES, internal oblique, and rectus abdominis muscle lower extremity isometric contraction in the supping position. In particular, the multifidus and erector spine muscle activity was significantly increased during hip flexion.

The results show that the contraction of the muscle's origin and insertion goes over the range and is transferred to other muscles, tendons, ligaments, fascia, joint capsule or skeletal, and that the upper and lower extremity muscle contraction can affect the contralateral trunk muscle activity [23]. The HAM is anatomically linked with the ligaments near the ligaments of the ischial tuberosity, which are connected to the ES muscles.

The sacral tuberous ligament is composed of three bands, which are the outer, inner, and upper fiber bands. Of these bands, the upper band is attached to the superficial component of the sacral iliac ligament and connects the coccyx and the posterior superior iliac spine. The biceps femoris and GM are associated with sacral tuberosity ligaments, which can increase the muscle contraction tension with lower limb extension [24]. Andry et al. [25] reported that the deep multifidus muscle adheres to the inner part of the upper sacroiliac ligament and that the contraction of the lower extremity muscle affects the muscle activity of the deep multifidus muscle. Therefore, increases in the nondominant side muscle activity is observed when performing the FTT and TT toe task positions, which leads to increases in muscle activity of the dominant side HAM activity. Also, increased dominant HAM and ES muscle activity is exhibited during the trunk extension period.

The limitations of this study are as follows. First, there is a limitation in generalizing the results of this study due to the small number of subjects. Second, although the subjects performed the three different motions in a random order, the learning effect could not be eliminated.

The purpose of this study was to analyze the interaction effect of the toe touch task posture on the trunk and lower limb muscle activity in healthy subjects and to provide basic data on the evaluation of the injuries and diseases that could change the lumbar pelvic rhythm. Further research is needed to further investigate the difference between the lumbar pelvic rhythm of hemiplegic patients and the general population.

\section{Conflict of Interest}

The authors declared no potential conflicts of interest with respect to the authorship and/or publication of this article.

\section{References}

1. Wolf S. The malalignment syndrome: diagnosis and treatment of common pelvic and back pain. Edinburgh: Churchill Livingstone; 2012.

2. Scholtes SA, Gombatto SP, Van Dillen LR. Differences in lumbopelvic motion between people with and people without low back pain during two lower limb movement tests. Clin Biomech (Bristol, Avon) 2009;24:7-12.

3. Lötters F, Burdorf A, Kuiper J, Miedema H. Model for the work-relatedness of low-back pain. Scand J Work Environ Health 2003;29:431-40.

4. Marras WS, Lavender SA, Leurgans SE, Fathallah FA, Ferguson SA, Allread WG, et al. Biomechanical risk factors for occupationally related low back disorders. Ergonomics 1995;38:377410

5. Xu Y, Bach E, Orhede E. Work environment and low back pain: the influence of occupational activities. Occup Environ Med 1997;54:741-5.

6. Ritvanen T, Zaproudina N, Nissen M, Leinonen V, Hänninen O. Dynamic surface electromyographic responses in chronic low back pain treated by traditional bone setting and conventional physical therapy. J Manipulative Physiol Ther 2007;30:31-7.

7. Rene C. Soft tissue pain and disability. Philadelphia, PA: F.A. Davis; 1996.

8. Leinonen V, Kankaanpää M, Airaksinen O, Hänninen O. Back and hip extensor activities during trunk flexion/extension: effects of low back pain and rehabilitation. Arch Phys Med Rehabil 2000;81:32-7.

9. Hodges PW, Richardson CA. Altered trunk muscle recruitment in people with low back pain with upper limb movement at different speeds. Arch Phys Med Rehabil 1999;80:1005-12.

10. Shan X, Wei Y, Chen Z, Fan L, Shi W, Yang S. Effect of leg support on muscle cross-correlation of bilateral erector spinae during trunk flexion-extension performance. Gait Posture 2014;39: 161-5.

11. Fieldman H. Relative contribution of the back and hamstring muscles in the performance of the toe-touch test after selected extensibility exercises. Res Q 1968;39:518-23.

12. Criswell E, Cram JR. Cram's introduction to surface electromyography. 2nd ed. Sudbury, MA: Jones and Bartlett; 2011.

13. Jo JY. Comparison of lower extremity muscle activation during forward step tasks on weight bearing and step length [Master thesis]. Seoul: Sahmyook University; 2017.

14. Boren K, Conrey C, Le Coguic J, Paprocki L, Voight M, Robinson TK. Electromyographic analysis of gluteus medius and gluteus maximus during rehabilitation exercises. Int J Sports Phys Ther 2011;6:206-23.

15. Bolgla LA, Uhl TL. Electromyographic analysis of hip rehabilitation exercises in a group of healthy subjects. J Orthop Sports Phys Ther 2005;35:487-94.

16. Perry J, Burnfield JM. Gait analysis: normal and pathological function. Thorofare: Slack; 2010.

17. Lyon IN, Day BL. Control of frontal plane body motion in human 
stepping. Exp Brain Res 1997;115:345-56.

18. Prince F, Winter DA, Archer SE. Assessment of postural control during quiet stance with different foot configurations. Gait Posture 1995;3:110.

19. Shumway-Cook A, Woollacott MH. Motor control: translating research into clinical practice. Philadelphia, PA: Lippincott Williams \& Wilkins; 2011.

20. Neumann DA, Kelly ER, Kiefer CL, Martens K, Grosz CM. Kinesiology of the musculoskeletal system: foundations for rehabilitation. 3rd ed. St. Louis, MO: Elsevier; 2016.

21. Woo YK, Park JW, Choi JD, Hwang JH, Kim YH. Electromyographic activities of lower leg muscles during static balance control in normal adults. Phys Ther Korea 2004;11:35-45.

22. Park HJ, Sim SM, Choi JD, Oh DW. The effects of isometric low- er limb contraction on the activation of contralateral trunk muscles in healthy young adults in supine position. Phys Ther Korea 2012;19:11-9.

23. Tarnanen SP, Ylinen JJ, Siekkinen KM, Mälkiä EA, Kautiainen HJ, Häkkinen AH. Effect of isometric upper-extremity exercises on the activation of core stabilizing muscles. Arch Phys Med Rehabil 2008;89:513-21.

24. Vleeming A, Schuenke MD, Masi AT, Carreiro JE, Danneels L, Willard FH. The sacroiliac joint: an overview of its anatomy, function and potential clinical implications. J Anat 2012;221: 537-67.

25. Andry V, Vert M, Rob S. Movement, stability and lumbopelvic pain: integration of research and therapy. 2nd ed. Edinburgh: Churchill Livingstone; 2007. 